\title{
Treatment of nasal microcystic adnexal carcinoma with an expanded rotational forehead skin flap: A case report and review of the literature
}

\author{
RONG-RONG ZHOU, QI-MING ZHAO, XU-DONG ZHANG and JING-BING GAN \\ Department of Plastic Surgery, The $117^{\text {th }}$ Hospital of People's Liberation Army, Hangzhou, Zhejiang 310013, P.R. China
}

Received June 4, 2014; Accepted May 27, 2015

DOI: $10.3892 /$ etm.2015.2614

\begin{abstract}
Microcystic adnexal carcinoma (MAC) is a rare and locally aggressive adenocarcinoma with low-grade malignancy. The present study describes the first reported case and treatment of a Chinese male with a MAC located on the nasal dorsum and nosewing. A 44-year-old man presented with a nasal deformity caused by local repeated infections following an accidental injury to the nose 20 years previously. The nose had been injured by a brick, and treatment at a local hospital 12 years previously had resulted in a nasal scar and a gradually enlarging mass. A physical examination revealed a hypertrophic deformity of the nose and an indurated scar plaque, measuring $2.0 \times 2.0 \mathrm{~cm}$, on the nasal dorsum and nosewing. Microscopic examination revealed a tumor consisting of solid cell nests and a cystic structure with a capsular space. In addition, ductal cells of an adnexal cell origin were visible in the outer epithelium. The medial portion exhibited a microductal structure and invasion of deeper tissues without evident atypia. The tumor cells presented normal nuclear to cytoplasmic ratios and minimal mitotic activity. Pathological examination verified that the tumor was a MAC of low-grade malignancy. A complete surgical resection was performed via Mohs micrographic surgery (MMS), and reconstruction was achieved using an expanded rotational forehead skin flap. No tumor recurrence was detected after a three-year follow-up period. Therefore, for effective treatment of similar MAC cases, complete surgical resection using MMS is recommended, and successful reconstruction may be achieved using an expanded skin flap.
\end{abstract}

\section{Introduction}

Microcystic adnexal carcinoma (MAC) is a rare, locally aggressive type of adenocarcinoma with low-grade malig-

Correspondence to: Dr Qi-Ming Zhao, Department of Plastic Surgery, The $117^{\text {th }}$ Hospital of People's Liberation Army, 14 Lingyin Road, Hangzhou, Zhejiang 310013, P.R. China

E-mail: zhaoqmzx@aliyun.com

Key words: microcystic adnexal carcinoma, Mohs micrographic surgery, rotational forehead skin flap nancy and extremely low metastatic ability (1-3). Since the first report of a MAC in 1982 by Goldstein et al (1), only 300 cases of MAC have been described to date. MACs are also known as sclerosing sweat duct carcinomas, eccrine epitheliomas and syringomatous carcinomas (4-6). MACs are most frequently located in the head and neck area (7); however, the occurrence of nasal MACs is extremely rare. MAC is usually diagnosed by detection of characteristic histological features (1-3). Histologically, MACs contain numerous keratin cysts, basaloid cells and squamous cell islands that form ductular and glandular structures, which often invade nerves $(2,3)$. These characteristics complicate the surgical treatment of MAC (8). There are several therapies to treat MAC, including standard excision (SE), Mohs micrographic surgery (MMS), irradiation and chemotherapy (3-6); however, there is no consensus regarding the treatment of MAC due to its rarity. Furthermore, there is no reported incidence rate of MAC since it is so rare.

In the present study, a novel case of a Chinese man with a MAC located on the nasal dorsum and nosewing is described. Mohs micrographic surgery (MMS) was performed to ensure the complete removal of tumorous tissue. In addition, successful reconstruction of the area was accomplished using an expanded rotational forehead skin flap, which restored the normal nose shape and skin color. There were no signs of tumor recurrence after a three-year follow-up period, indicating the effectiveness of MMS for the treatment of MAC.

\section{Case report}

Patient information. A 44-year-old Chinese man was admitted to the $117^{\text {th }}$ Hospital of People's Liberation Army (Hangzhou, China) following repeated local infections to his nose. An initial injury had occurred 20 years previously when the nose was subjected to blunt trauma inflicted by a brick. Treatment at a local hospital 12 years prior had resulted in a nasal scar and a gradually enlarging mass. Aside from this complaint, the patient exhibited a normal general condition.

A physical examination revealed a hypertrophic deformity of the nose and an indurated scar plaque, measuring $2.0 \times 2.0 \mathrm{~cm}$, located on the nasal dorsum and nosewing, with no redness, pain, ulceration or scaling (Fig. 1). Compared with the surrounding skin, the plaque was raised and slightly lighter in color. The nasal cavity sustained normal ventilation and olfactory sensation, without purulent discharge or nasal 


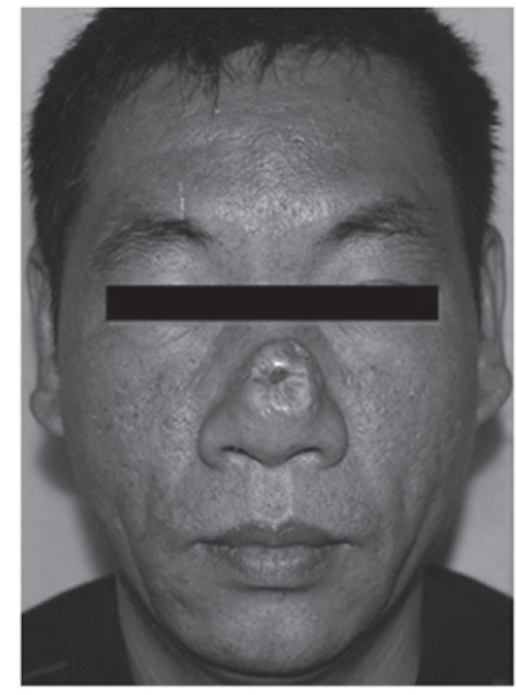

Figure 1. Macroscopic appearance of the tumor. The patient presented with a tumor that was similar in appearance to a depressed scar without ulceration or clinical symptoms.

septum deviation. No lymphadenopathy was observed, and routine laboratory examinations (including routine blood and urine tests, biochemical, electrocardiographic, chest x-ray, and abdominal $\mathrm{B}$ ultrasonic examinations) produced normal results. The patient had no significant medical history, no known incidence of similar disease in his family history and had no known exposure to radiation or chemicals. The patient provided informed consent, and the study was approved by the Ethics Committee of the $117^{\text {th }}$ Hospital of People's Liberation Army.

Surgical methods. A forehead tissue expander (Kidney type, $100 \mathrm{ml}$; Shanghai Winner Plastic Surgery Products Co., Ltd, Shanghai, China) was implanted under local anesthesia (lidocaine hydrochloride, Shanghai Zhaohui Pharmaceutical Co. Ltd., Shanghai, China; Fig. 2). The expander was inflated with saline (Hunan Kelun Pharmaceutical Co. Ltd., Yueyang, China) every 4-7 days for 2 months, until a final volume of $\sim 132 \mathrm{ml}$ was reached. Following the final filling of the expander, the expander was removed and an extensive resection of the tumor was performed. A $0.5-\mathrm{cm}$ tumor-free margin surrounding the skin lesion was identified and excised using MMS (9). Immediate reconstruction was performed using an expanded rotational forehead skin flap (Figs. 2 and 3). Two months after the reconstruction, the flap was separated from its pedicle (Fig. 4). The stitches (model no. 0) were removed the following week, and the patient was discharged from hospital. The reconstruction of the defect maintained normal nasal function and improved esthetics (Fig. 5). No recurrence was detected during a three-year follow-up period.

Histology. Macroscopic observations revealed that the tumor was round and rough, with beige coloring and a diameter of $\sim 2.5 \mathrm{~cm}$. Microscopic examination (Olympus microscope BX43; Olympus, Tokyo, Japan) demonstrated a heterogeneous tumor cell composition with multiple differentiations. The external portion of the tumor consisted of solid cell nests (Fig. 6A) and cystic structures with capsular spaces
(Fig. 6B), in addition to ductal cells of an adnexal cell origin. The medial portion of the tumor consisted of microductal structures formed by ductal epithelial cells, which invaded the deeper tissues without evident atypia (Fig. 6C). The tumor cells presented a normal nuclear to cytoplasmic ratio and minimal mitotic activity (Fig. 6D). Furthermore, the tissue in the deepest region of the tumor exhibited interstitial sclerosis and collagenization (Fig. 6E). Immunohistochemical analysis revealed positive staining for epithelial membrane antigen (EMA; MAB-0581, Fuzhou Maixin Biotechnology Development Co. Ltd., Fuzhou, China), cytokeratin (CK; MAB-0049, Fuzhou Maixin Biotechnology Development Co. Ltd.), B-cell lymphoma 2 (Bcl-2; MAB-0014, Fuzhou Maixin Biotechnology Development Co. Ltd.) and S-100 proteins (MAB-0585, Fuzhou Maixin Biotechnology Development Co. Ltd.), with negative staining for CK7 (kit-0021, Fuzhou Maixin Biotechnology Development Co. Ltd.), CK20 (MAB-0057, Fuzhou Maixin Biotechnology Development Co. Ltd.) and smooth muscle actin (SMA; MAB-0575, Fuzhou Maixin Biotechnology Development Co. Ltd.) proteins. Based on these results, a diagnosis of MAC (low-grade malignancy) was confirmed.

\section{Discussion}

Although the etiology of MACs is unclear, previous studies have reported an association between MACs and exposure to radiation $(2,10)$. MACs generally affect the lips and facial regions in adult patients, and are more prevalent among females. The appearance of a MAC is usually similar to a depressed scar with no clinical symptoms, but may present with occasional swelling and numbness, and rarely producing ulcerations (1-3). These tumors are able to develop gradually for a number of months or years in a locally aggressive mode. MACs have a $50 \%$ postoperative local recurrence rate, with very rare instances of metastasis $(1,2,11)$. The macroscopic characteristics of the MAC case reported in the present study were similar to those reported in previous literature $(2,3,7,9,10)$. Since there was no incidence of similar disease in the patient's family history and no radioactive or chemical exposure, the cause of the disease was presumed to be associated with the repeated local infections.

A diagnosis of a MAC is usually established based on the detection of characteristic histological features $(1-3,12)$. Microscopic examinations show numerous keratocysts above the dermis layer of the tumor. In addition, the tumor cells are basal-like, and certain tumor cells have transparent cytoplasm, forming squamous cell clusters, island or cords, or are arranged in a grid. Furthermore, in the medial portion of the tumor, there are ductular and glandular structures. Mitotic structures are rare in these tumor cells, and they lack significant atypia. MAC tumor cells invade the whole dermis, frequently invading the striated muscle, nerves, vascular adventitia, cartilage tissue and periosteum $(1-3,13)$. Although MACs have the ability to locally invade tissues, very few cases have reported systemic metastasis and mortality. In the present case, the histological characteristics of the MAC were similar to those detected in previous studies $(1,4,7,8)$.

Although a diagnosis of MAC may be confirmed based on the detection of certain histological characteristics, 


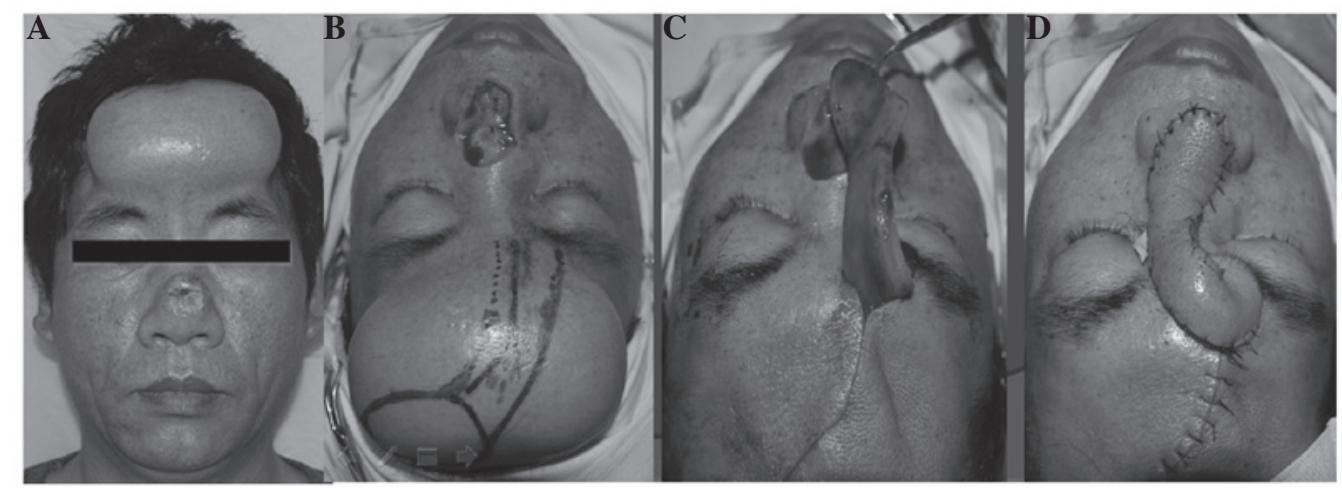

Figure 2. Generation of an expanded forehead skin flap. (A) A forehead tissue expander was implanted; (B) extensive resection of the tumor was conducted; (C and D) reconstruction was performed using the expanded rotational forehead skin flap.

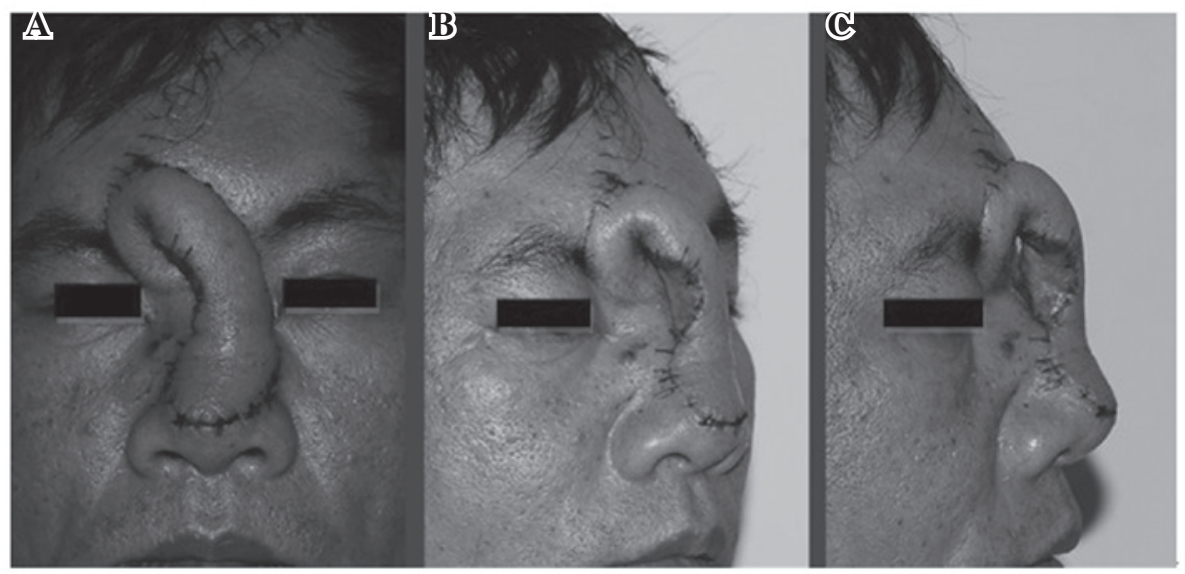

Figure 3. Reconstruction with the expanded forehead skin flap, as shown from the (A) anterior position, (B) right anterior oblique position and (C) right lateral position.

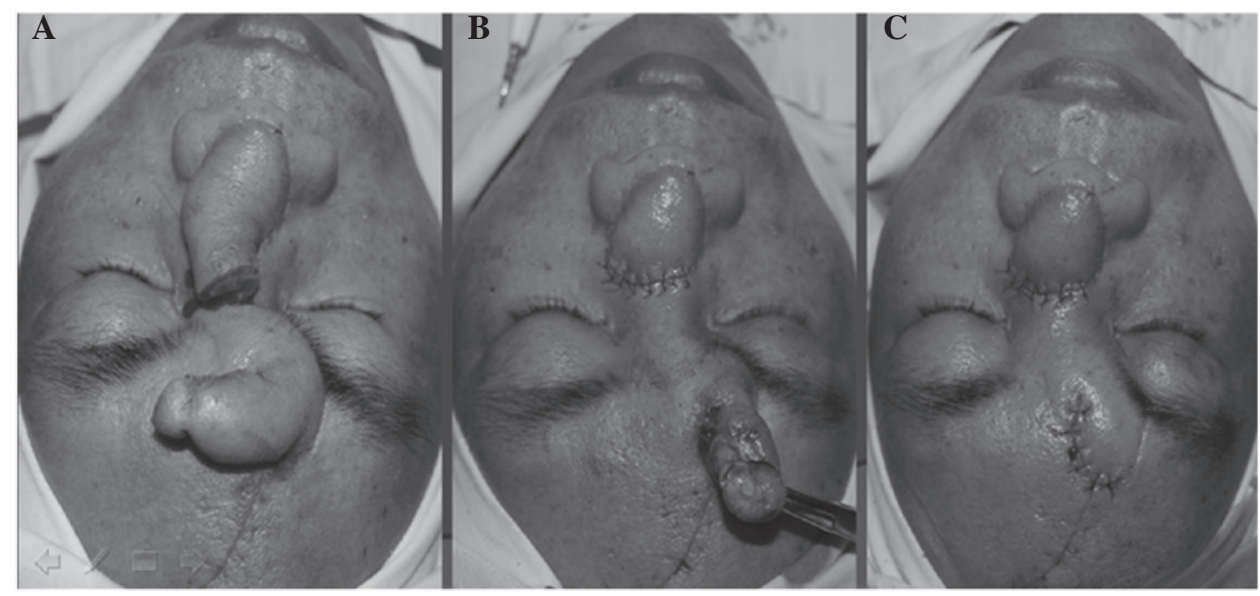

Figure 4. Flap separation. (A) Abscise of the flap at the distal; (B) suture of the flap incision at the nasal root; (C) suture of the flap incision at the forehead.

immunohistochemical confirmation may be a useful ancillary diagnostic tool. In the present case, immunohistochemical staining was positive for EMA, CK, Bcl-2 and S-100 proteins, and negative for CK20, CK7 and SMA. By contrast, in the study by Smith et al (14), 10 MAC cases were analyzed and all the MAC tumor cells were shown to exhibit a distinctive pattern of staining for CK7 and $\alpha$-SMA, in addition to CD34,
EMA, Ber-EP4 and S-100 proteins. However, the tumor cells were negative for c-erbB-2. In the present case, $<2 \%$ of the tumor cells were Ki-67-positive, which was in accordance with the results of Smith et al (14), who found that $<5 \%$ of tumor cells were Ki-67-positive in MAC. These results indicate that the low level of $\mathrm{Ki}-67$ reflects the low proliferative rate, while the additional immunohistochemical markers 


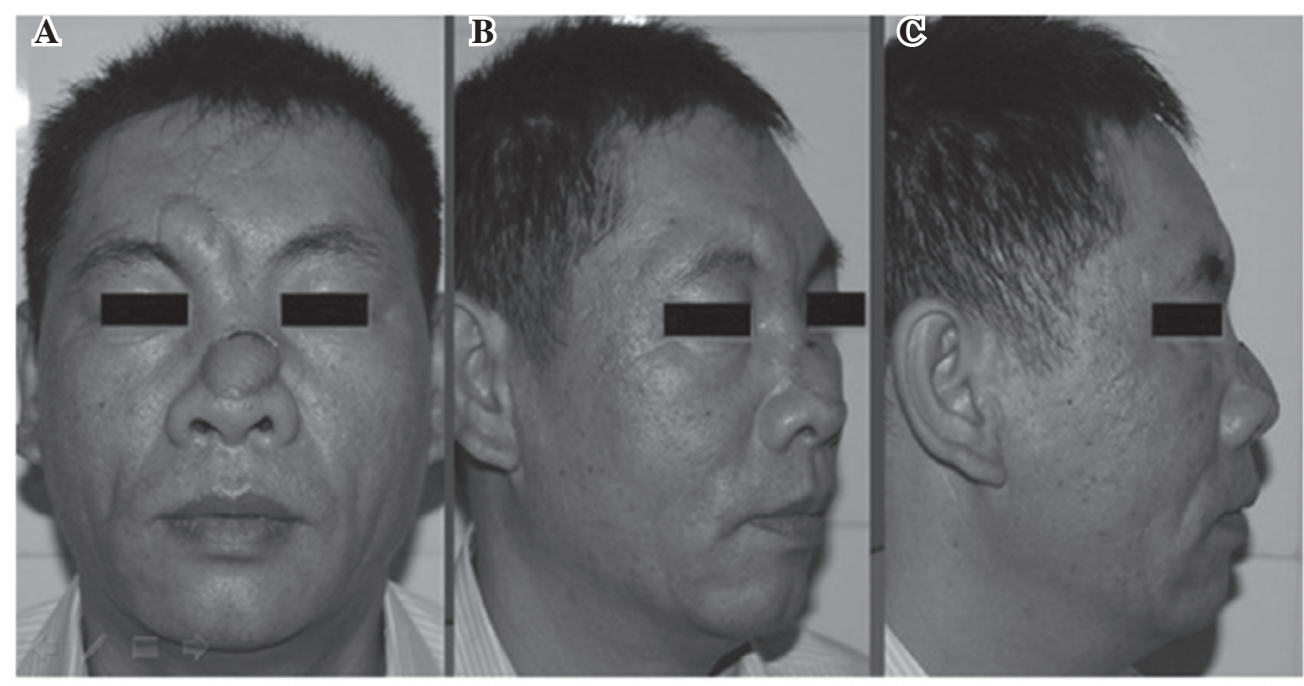

Figure 5. Appearance of the reconstruction following removal of the stitches from an (A) anterior position, (B) right anterior oblique position and (C) right lateral position. Reconstruction of the defect resulted in normal function and esthetics.
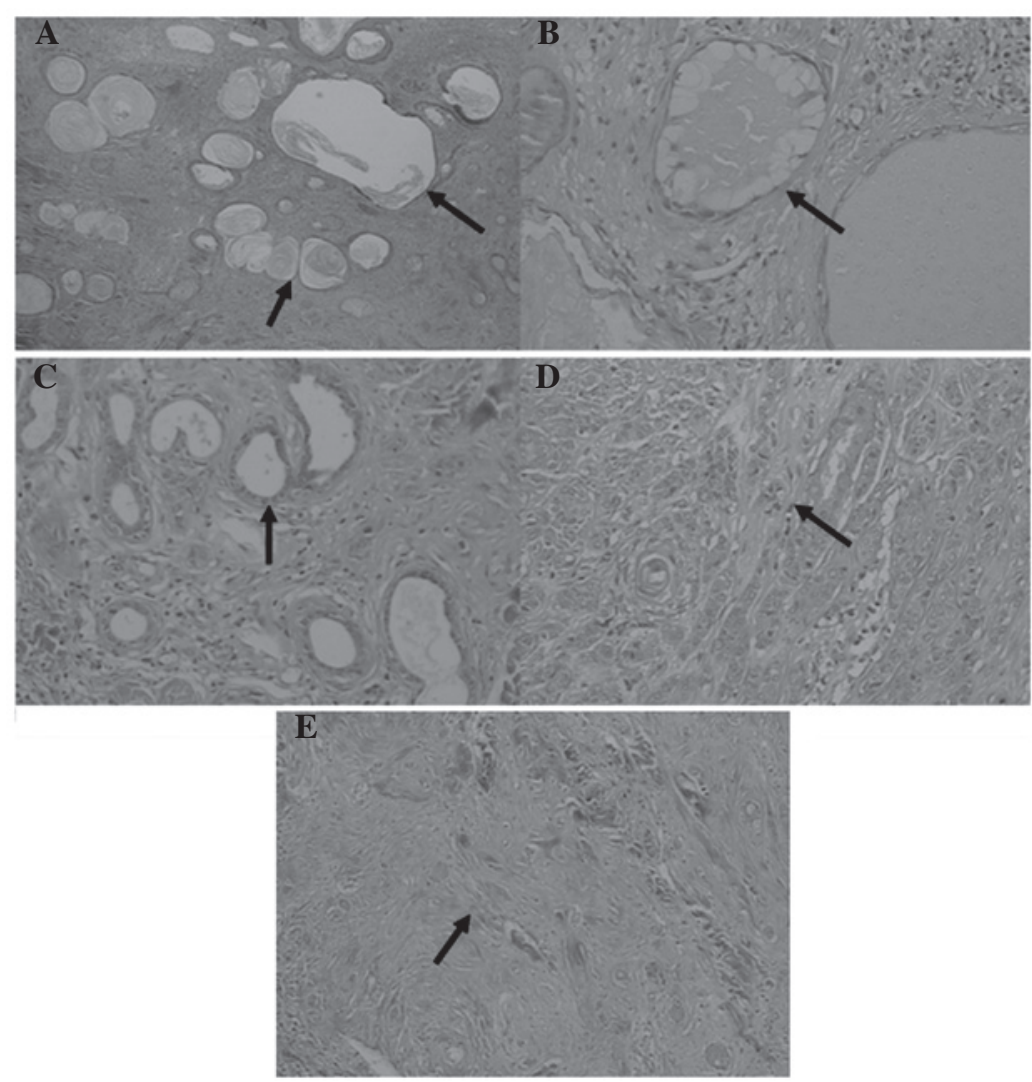

Figure 6. Histopathological observations. (A) Exterior portion of the tumor exhibited solid cell nests and cystic structures (shown by arrows) with a capsular space (magnification, x40). (B) Ductal cells (shown by arrows) were visible in the exterior portion, appearing as clear cells originating from adnexal cells (magnification, x200). (C) Medial tumor portion comprised a microductal structure (as shown by arrow) formed by ductal epithelial cells, with a tendency to invade the deeper tissues without evident atypia (magnification, x200). (D) Tumor cells in the medial portion exhibited a normal nuclear to cytoplasmic ratio (as shown by arrow), and minimal mitotic activity (magnification, x200). (E) Deeper tumor tissue exhibited marked interstitial sclerosis and collagenization (as shown by arrow; magnification, $\mathrm{x} 100$ ).

support the divergent patterns of adnexal differentiation in MAC. Additionally, Hoang et al (15) reported that CK15 was a useful marker for distinguishing MAC from cases of infiltrative basal cell carcinoma and squamous cell carcinoma with ductal differentiation. However, further studies are required to identify a reliable immunohistochemical marker specific to MACs.

Therapy for MACs remains a challenge, as the tumor-free margin is significantly more extensive than can be determined clinically and the tumors exhibit a marked tendency 
for perineural invasion (3-5). Numerous treatment modalities have been used to date, including standard excision (SE), MMS, irradiation and chemotherapy $(6,16)$; however, a number of studies have found that MAC is not sensitive to radiotherapy and chemotherapy $(5,17)$. Although the extent of resection using SE is reduced and the technique results in improved esthetics, the tumor recurrence rate is higher. Chiller et al (18) observed that $30 \%$ of MAC patients that initially underwent SE treatment were required to submit to SE or MMS at least once again, since the margins of the specimens were positive for tumor cells. In addition, Chow et al (19) reported that $40-60 \%$ of patients experienced one or more local recurrences following standard wide local excision.

MMS is a therapeutic approach for skin cancer removal that aims to achieve the highest possible cure rates, while minimizing the size of the wound and consequent distortions. MMS involves the removal of the tumor in stages by histologically confirming clear margins on frozen sections and by treating the resultant defect. A number of studies have been published regarding the treatment of MACs with MMS. Chiller et al (18) observed that the size of the affected surface area after full excision with MMS was four times larger compared with the clinically apparent size. Therefore, this method ensures complete excision of the cancerous tissue, and subsequently the recurrence rates following MMS are low $(0-12 \%)(11)$. In the present study, the patient was treated with MMS following reconstruction, and no tumor recurrence was detected during the three-year follow-up period.

These results indicate that the therapeutic and esthetic advantages of MMS may enable the surgical technique to become a useful therapeutic option for the complete eradication of MAC. However, the repair of the resulting defect is a considerable reconstructive challenge, particularly in the centrofacial region. In the present study, a $0.5-\mathrm{cm}$ tumor-free margin surrounding the skin lesion was identified using MMS and the contained area was excised, with the incision depth reaching into the deep fasciae. The defect was too large to directly suture or repair with a local flap. Therefore, an innovative reconstruction approach was attempted using an expanded rotational forehead skin flap, which resulted in normal function and esthetics. Based on the present case, a key factor for successful reconstruction is the full expansion of the skin flap with a sufficient volume of saline. A similar procedure was reported by Rustemeyer et al (20), who used a rotational flap from the cheek and lower lip in combination with an Abbe flap for philtrum and vermilion reconstruction, which resulted in a successful reconstruction following the complete resection of the MAC.

In conclusion, the present study was the first to report the case of a Chinese man with a MAC located on the nasal dorsum and nosewing. For similar cases of MAC, complete surgical resection with MMS is recommended as the most effective therapeutic option. An expanded rotational forehead skin flap may be used for reconstruction to achieve normal function and esthetics.

\section{Acknowledgements}

The authors thank the Ethics Committee of the $117^{\text {th }}$ Hospital of People's Liberation Army for their assistance.

\section{References}

1. Goldstein DJ, Barr RJ and Santa Cruz DJ: Microcystic adnexal carcinoma: A distinct clinicopathologic entity. Cancer 50: 566-572, 1982.

2. Yu JB, Blitzblau RC, Patel SC, Decker RH and Wilson LD: Surveillance, Epidemiology, and End Results (SEER) database analysis of microcystic adnexal carcinoma (sclerosing sweat duct carcinoma) of the skin. Am J Clin Oncol 33: 125-127, 2010.

3. Bier-Laning cm, Hom DB, Gapany M, Manivel JC and Duvall AJ III: Microcystic adnexal carcinoma: Management options based on long-term follow-up. Laryngoscope 105: 1197-1201, 1995.

4. Lipper S and Peiper SC: Sweat gland carcinoma with syringomatous features: A light microscopic and ultrastructural study. Cancer 44: 157-163, 1979.

5. Glatt HJ, Proia AD, Tsoy EA, Fetter BF, Klintworth GK, Neuhaus R and Font RL: Malignant syringoma of the eyelid. Ophthalmology 91: 987-990, 1984.

6. Alessi E and Caputo R: Syringomatous carcinoma of the scalp presenting as a slowly enlarging patch of alopecia. Am J Dermatopathol 15: 503-505, 1993.

7. Beltramini GA, Baj A, Moneghini L, Poli T, Combi VA and Giannì $\mathrm{AB}$ : Microcystic adnexal carcinoma of the centrofacial region: A case report. Acta Otorhinolaryngol Ital 30: 213, 2010.

8. Wetter R and Goldstein GD: Microcystic adnexal carcinoma: A diagnostic and therapeutic challenge. Dermatol Ther 21: 452-458, 2008.

9. Leibovitch I, Huilgol SC, Selva D, Lun K, Richards S and Paver R: Microcystic adnexal carcinoma: Treatment with Mohs micrographic surgery. J Am Acad Dermatol 52: 295-300, 2005.

10. Abbate M, Zeitouni NC, Seyler M, Hicks W, Loree T and Cheney T: Clinical course, risk factors, and treatment of microcystic adnexal carcinoma: A short series report. Dermatol Surg 29: 1035-1038, 2003.

11. Snow S, Madjar DD, Hardy S, Bentz M, Lucarelli MJ, Bechard R, Aughenbaugh W, McFadden T, Sharata H, Dudley C, et al: Microcystic adnexal carcinoma: Report of 13 cases and review of the literature. Dermatol Surg 27: 401-408, 2001.

12. Sirikanjanapong S, Seymour AW and Amin B: Cytologic features of microcystic adnexal carcinoma. Cytojournal 8: 5, 2011.

13. Hamsch $\mathrm{C}$ and Hartschuh W: Microcystic adnexal carcinoma - aggressive infiltrative tumor often with innocent clinical appearance. J Dtsch Dermatol Ges 8: 275-278, 2010 (In English and German).

14. Smith KJ, Williams J, Corbett D and Skelton H: Microcystic adnexal carcinoma: An immunohistochemical study including markers of proliferation and apoptosis. Am J Surg Pathol 25: 464-471, 2001.

15. Hoang MP, Dresser KA, Kapur P, High WA and Mahalingam M: Microcystic adnexal carcinoma: An immunohistochemical reappraisal. Mod Pathol 21: 178-185, 2008.

16. Pugh TJ, Lee NY, Pacheco T and Raben D: Microcystic adnexal carcinoma of the face treated with radiation therapy: A case report and review of the literature. Head Neck 34: 1045-1050, 2012.

17. Stein JM, Ormsby A, Esclamado R and Bailin P: The effect of radiation therapy on microcystic adnexal carcinoma: A case report. Head Neck 25: 251-254, 2003.

18. Chiller K, Passaro D, Scheuller M, Singer M, McCalmont T and Grekin RC: Microcystic adnexal carcinoma: Forty-eight cases, their treatment, and their outcome. Arch Dermatol 136: $1355-1359,2000$.

19. Chow WC, Cockerell CJ and Geronemus RG: Microcystic adnexal carcinoma of the scalp. J Dermatol Surg Oncol 15: 768-771, 1989.

20. Rustemeyer J, Zwerger S, Pörksen M and Junker K: Microcystic adnexal carcinoma of the upper lip misdiagnosed benign desmoplastic trichoepithelioma. Oral Maxillofac Surg 17: 141-144, 2013. 